First publ. in: Optics Communications 174 (2000), 1-4, pp. 145-149

\title{
Nanosecond time-resolved reflectivity determination of the melting of metals upon pulsed laser annealing
}

\author{
J. Boneberg * , J. Bischof, P. Leiderer \\ Universität Konstanz, 78467 Konstanz, Germany
}

Received 4 August 1999; accepted 22 October 1999

\begin{abstract}
Thin metal films $(\mathrm{Cu}, \mathrm{Au}, \mathrm{Ni})$ on quartz glass were illuminated with nanosecond pulses of a frequency doubled Nd:YAG laser $(\lambda=532 \mathrm{~nm})$. The transient reflectivity behaviour was probed at the wavelength $633 \mathrm{~nm}$, where these metals exhibit Drude-like optical behaviour: the reflectivity decreases with temperature in the solid and in the liquid state as well as at the solid-liquid phase transition. It is shown that nanosecond time-resolved reflectivity measurements can be used to determine the melting of these metal surfaces due to the reflectivity difference between the solid and the liquid phase.
\end{abstract}

PACS: 42.62. - b; 78.47.+ p; 78.90.+t; 81.40.Wx

Keywords: Laser-annealing; Metal; Melting; Optical properties

During the last years the possible application fields of pulsed laser irradiation exhibited a continuous increase. Some examples are material modifications like cutting, drilling, alloying as well as cleaning and ablation. In many technical applications metallic surfaces have to be modified. In special cases it might be worth achieving a real time control of the condition of the metallic surface during the modification. A possible approach to this task are in situ time-resolved reflectivity measurements at the metal surface. On semiconductor surfaces numerous measurements of this kind have been performed especially on silicon, e.g. Ref. [1]. On metal surfaces, however, only few experimental studies have

Corresponding author. Tel.: +49-7531-882-256; fax: +497531-883-127; e-mail: johannes.boneberg@uni-konstanz,de been published up to now [2-6]. Most of these arose in the context of transient resistivity measurements of structured thin metallic films. While these electrical data were analysed quantitatively, the optical data have been used in a qualitative way only. This might be due to the fact that the expected reflectivity changes on metals are small compared to semiconductors. As an example the reflectivity of Si changes from $32 \%$ to $70 \%$ upon melting ( $\lambda=633 \mathrm{~nm}$, normal incidence) as a consequence of the phase transition from the solid which is a semiconductor to the liquid with metallic behaviour [7]. In comparison, on a metal like $\mathrm{Ni}$ a change of only $2 \%$ can be expected as calculated from the optical constants [8]. In this paper we describe time-resolved reflectivity measurements in detail and demonstrate that despite the small reflectivity changes the phase transition can clearly be resolved. As will be shown for several 


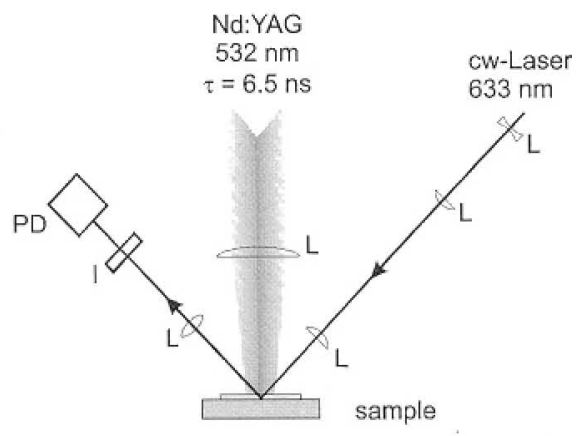

Fig. 1, Experimental setup: L: lens, I: interference filter, PD: photodiode.

metals (Au, Ni, Cu) time-resolved reflectivity studies allow an in situ control of the melting of the surface.

The annealing laser was a frequency-doubled Qswitched Nd:YAG laser $(\lambda=532 \mathrm{~nm})$ with a pulse length of $7 \mathrm{~ns}$ (full width at half maximum) and a beam profile close to $\mathrm{TEM}_{00}$. The Nd:YAG pulse, incident nearly perpendicular to the surface (Fig. 1), was only mildly focused to a spot diameter of about $0.5 \mathrm{~mm}$. For the measurement of the reflectivity $R$ we used an $s$-polarized continuous wave $\mathrm{He}-\mathrm{Ne}$ laser $(10 \mathrm{~mW}, 633 \mathrm{~nm})$ with an angle of incidence of $45^{\circ}$ with respect to the surface normal. The laser was focused to a $1 / \mathrm{e}$ diameter of below $15 \mu \mathrm{m}$ at the surface, so that the variation of the pulse laser beam intensity across the diameter of the test laser could be neglected. The specularly reflected light was detected by a fast pin diode (FND 100) and registered by a fast digital storage oscilloscope (HP54111D). If necessary, a $1 \mathrm{GHz} \mathrm{AC}$ amplifier was inserted in addition. In that case the absolute values of $R$ were determined before and after a laser pulse. Altogether the time resolution of the measurement was $1 \mathrm{~ns}$. The resolution in the reflectivity was determined to be $0.2 \%$. Interference filters in front of the pin diodes suppressed any contribution of the laser pulse to the measured signal.

Our samples were thermally evaporated metal films on quartz glass substrates. The thickness of the metal films, which was controlled by a quartz microbalance during the evaporation process in a $\mathrm{HV}$ chamber was in the range of $30-40 \mathrm{~nm}$. In order to enhance the adhesion of the metal layers, the substrates were first etched with argon ions and then a 1 $\mathrm{nm}$ Cr film was deposited onto the substrate before the evaporation of the actual sample layer.

In order to receive an impression of the expected reflectivity changes we calculated the reflectivity values for the metals with a computer programme for thin film optics on the basis of the optical constants as determined by Otter [8] for single crystals. Fig. 2 shows the calculated reflectivity for $s$-polarized light at an incident angle of $45^{\circ}$ as a function of temperature for the example $\mathrm{Cu}$. The reflectivity decreases almost linearly with increasing temperature at a slope of around $0.6 \%$ per $100 \mathrm{~K}$. The reflectivity difference for the solid phase between room temperature and the melting temperature $\Delta R_{\mathrm{RT}-\mathrm{MT}}$ amounts to about $6 \%$. Upon melting a further reflectivity decrease $\Delta R_{\mathrm{sl}}\left(T_{\mathrm{m}}\right)$ of $4.1 \%$ is expected. In order to determine the recrystallization velocity from the measurements we further determined the change of reflectivity at the melting temperature as function of the thickness of the molten $\mathrm{Cu}$ layer (Fig. 3). The reflectivity decreases continuously from the value of the solid state $(92.7 \%)$ towards the value of the liquid state $(88.6 \%)$. The total reflectivity change upon melting $\Delta R_{\mathrm{sl}}$ is $4.1 \%, 50 \%$ of this change is reached at a liquid layer thickness of $8 \mathrm{~nm}$.

Fig. 4 shows the measured time-resolved reflectivity of a $40 \mathrm{~nm} \mathrm{Cu}$ film during pulse laser annealing at energy densities of 70,115 , and $320 \mathrm{~mJ} / \mathrm{cm}^{2}$. The laser pulse is schematically depicted in the lower trace of Fig. 4(a). Before the application of the laser pulse the measurement yields a reflectivity

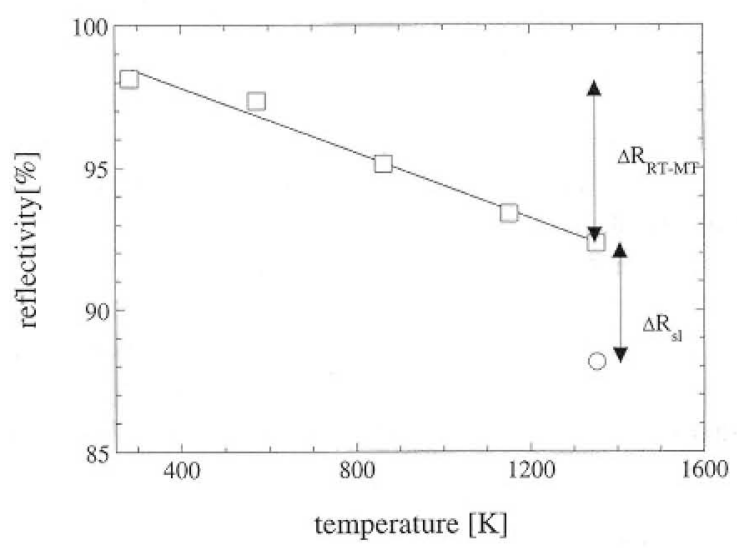

Fig. 2. Calculated reflectivity behaviour of a $\mathrm{Cu}$ thin film using the data of Otter [8]. 


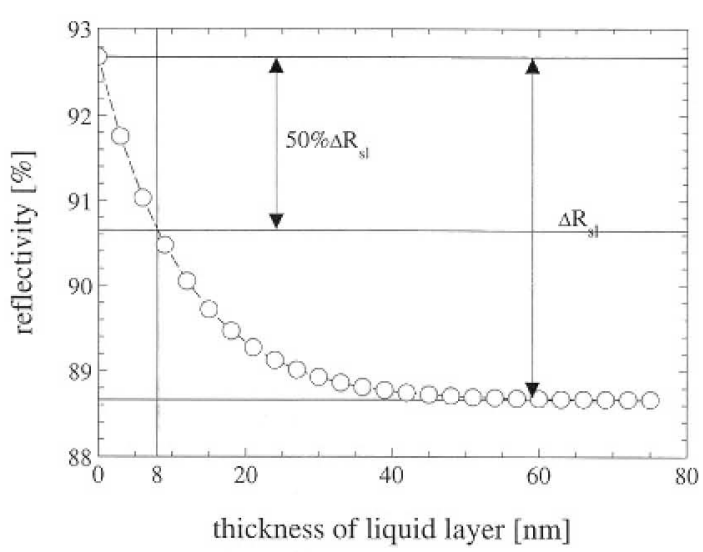

Fig. 3. Calculated reflectivity behaviour as function of liquid film thickness at $T_{\mathrm{m}}$.

(90.5\%) which is several percent below the value expected from the calculations. We attribute this difference to the slightly different values of the optical constants of the evaporated thin film compared to the single crystals which have been used by Otter [8].

At an energy density of $70 \mathrm{~mJ} / \mathrm{cm}^{2}$ (Fig. 4(a)) the reflectivity drops during the laser pulse from the starting value of $90.5 \%$ towards a minimum of $86.5 \%$ and then increases again towards the starting value. The interpretation of this behaviour is straightforward: Due to the absorbed energy the temperature of this surface layer increases. As a consequence the collision frequency increases which gives rise to a reflectivity decrease, in agreement with both a simple Drude model and the temperature dependent data of Otter. As a first approximation we can use the slope of the data of Fig. 2 to determine the maximum temperature increase from these data, which is estimated to $670 \mathrm{~K}$.

In Fig. 4(b) the energy density was increased to $115 \mathrm{~mJ} / \mathrm{cm}^{2}$. The transient reflectivity decrease is more pronounced now and amounts to $9.7 \%$, which is more than the expected reflectivity change upon heating up to the melting point. Nevertheless there is no clear indication of melting in this trace.

At a further enhanced energy density (Fig. 4(c)) a new feature appears in the reflectivity behaviour. First $R$ drops towards $75 \%$, and then increases again, but now displaying a plateau at $81 \%$. After 140 ns the slope of the reflectivity is increased during $26 \mathrm{~ns}$ before the starting value of $R$ is slowly reapproached on a timescale of $900 \mathrm{~ns}$. We interpret this time dependence in the following way: At such a high energy density the temperature in the probed surface layer increases well above the melting point during the laser pulse, therefore the reflectivity decreases below the liquid reflectivity at $T_{\mathrm{m}}, R_{\mathrm{l}}\left(T_{\mathrm{m}}\right)$. Heat transport into the substrate reduces the temperature in the film, whereas the temperature in the substrate increases. Therefore the temperature gradient into the substrate and thus also the heat transport are decreasing with time. Then (as we know from reflectivity measurements of the back side of the film, which are not shown here) around $t=130 \mathrm{~ns}$ solidification of the liquid film begins at the metal-substrate interface, and latent heat is released which compensates the heat loss by heat transport into the substrate in such a way that the temperature remains slightly below $T_{\mathrm{m}}$. Consequently the temperature at the film surface and the reflectivity are almost constant in this regime, which can be used to determine $R_{j}\left(T_{\mathrm{m}}\right)$. The solidification proceeds then towards the surface
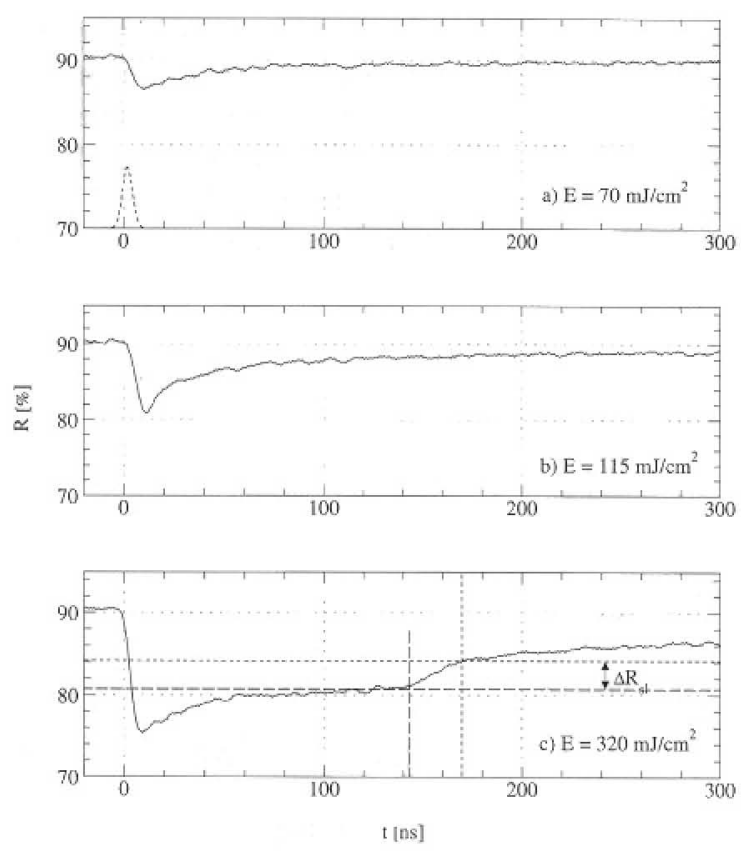

Fig. 4. Time-resolved reflectivity traces upon annealing of a 40 $\mathrm{nm} \mathrm{Cu}$ film at three different energy densities: (a) $70 \mathrm{~mJ} / \mathrm{cm}^{2}$; (b) $115 \mathrm{~mJ} / \mathrm{cm}^{2}$; and (c) $320 \mathrm{~mJ} / \mathrm{cm}^{2}$. 
and reaches the probed layer thickness at $140 \mathrm{~ns}$. As the solidification front passes the penetration depth of the test laser during the next $26 \mathrm{~ns}$, the reflectivity changes from $R_{1}\left(T_{\mathrm{m}}\right)$ to $R_{\mathrm{s}}\left(T_{\mathrm{m}}\right)$, the reflectivity of the solid state at $T_{\mathrm{m}}$. Thus the increase of reflectivity in this time interval indicates the solidification process. In this way one gets both $R_{\mathrm{l}}\left(T_{\mathrm{m}}\right)$ and $R_{\mathrm{s}}\left(T_{\mathrm{m}}\right)$ from a time-resolved measurement at enhanced energy density. The slow subsequent reflectivity increase of trace 4(c) (compared to $4(\mathrm{a})$ and $4(\mathrm{~b})$ ) is a result of the reduced thermal heat conduction. In comparison with Fig. 3 it is possible to determine the resolidification velocity: the first $50 \%$ of the increase of $\Delta R_{\mathrm{sl}}$ upon recrystallisation takes place in $10 \mathrm{~ns}$, corresponding to a resolidification velocity of 0.8 $\mathrm{m} / \mathrm{s}$.

A second example, given in Fig. 5 shows the reflectivity behaviour of a $47 \mathrm{~nm}$ Au film during pulsed laser illumination at energy densities of 170 and $320 \mathrm{~mJ} / \mathrm{cm}^{2}$. The absolute values both in energy density and in the absolute reflectivity changes differ from the first example. Nevertheless the same qualitative behaviour as in Fig. 4 can be observed: At the lower energy density the reflectivity decreases rapidly during the laser pulse and increases then towards the starting value. At the higher energy density once again a plateau appears. As before the resolifidication process can be identified by the steep increase of the reflectivity at the end of the plateau, here at 185 ns. The difference between liquid $\left(R_{\mathrm{l}}\left(T_{\mathrm{m}}\right)\right)$ and solid $\left(R_{\mathrm{s}}\left(T_{\mathrm{m}}\right)\right)$ reflectivity at the melt-

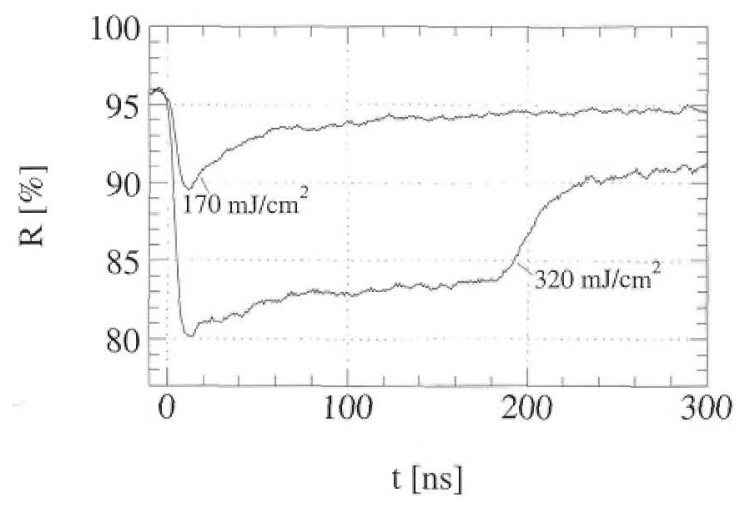

Fig. 5. Time-resolved reflectivity traces upon annealing of a 47 $n \mathrm{~m} \mathrm{Au}$ film at two different energy densities: (a) $170 \mathrm{~mJ} / \mathrm{cm}^{2}$; and (b) $320 \mathrm{~mJ} / \mathrm{cm}^{2}$.

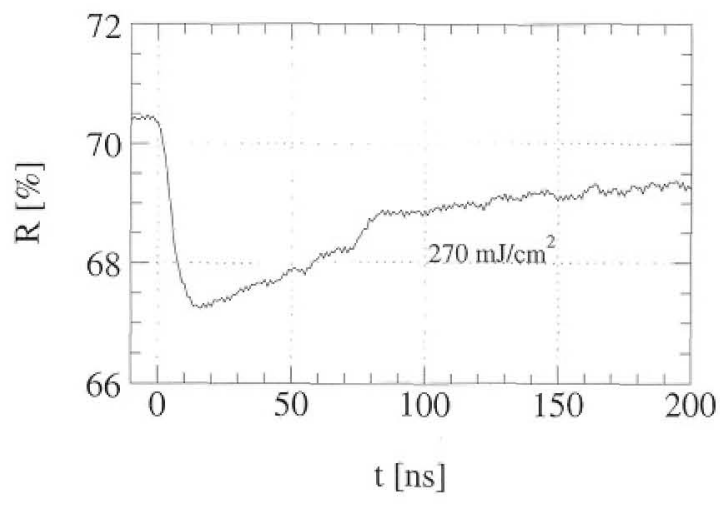

Fig. 6. Time-resolved reflectivity trace upon annealing of a $35 \mathrm{~nm}$ $\mathrm{Ni}$ film at the energy density $270 \mathrm{~mJ} / \mathrm{cm}^{2}$.

ing point is even more pronounced $\left(\Delta R_{s 1}\left(T_{m}\right)=6 \%\right)$ than for $\mathrm{Cu}$.

In the case of $\mathrm{Ni}$ the reflectivity changes are less marked, therefore the determination of $R_{\mathrm{I}}\left(T_{\mathrm{m}}\right)$ and $R_{\mathrm{s}}\left(T_{\mathrm{m}}\right)$ requires a higher sensitivity. Fig. 6 shows the result for an energy density of $270 \mathrm{~mJ} / \mathrm{cm}^{2}$. Once again the solidification (at $t=80 \mathrm{ns)}$ can clearly be identified although the difference between $R_{1}\left(T_{\mathrm{m}}\right)$ and $R_{\mathrm{s}}\left(T_{\mathrm{m}}\right)$ is below $0.5 \%$ in this case.

It has to be mentioned that the most critical experimental requirement for the identification of the resolidification is a constant energy density across the surface area probed by the test laser. Already a slight variation in energy density of the laser pulse shifts the phase transition in time. Therefore the increase between $R_{l}\left(T_{m}\right)$ and $R_{s}\left(T_{m}\right)$ is spread out in time. As a consequence the difference in the slope between the phase transition and the reflectivity change due to the cooling may be hidden due to intensity fluctuations of the test laser. Thus the identification of the phase transition may become difficult.

In conclusion we have shown that time-resolved reflectivity measurements allow the clear identification of the liquid-solid phase transition at metal surfaces in nanosecond pulsed laser annealing experiments. It will be interesting to study possible undercooling effects of metallic liquids after pulse laser melting with this technique. Furthermore electron and ion beam annealing experiments might benefit from time-resolved informations. 


\section{Acknowledgements}

We gratefully acknowledge financial support by the Deutsche Forschungsgemeinschaft (LE 315/15).

\section{References}

[1] D.H. Auston, C.M. Surko, T.N.C. Venkatesan, R.E. Slusher, J.A. Golovehenko, Appl. Phys. Lett. 33 (1978) 437.
[2] A.M. Bonch-Bruevich, Ya.A. Imas, G.S. Romanov, M.N. Libenson, I. Maltsev, Sov. Phys. Tech. Phys. 13 (1968) 640.

[3] T.E. Zavecz, M.A. Saifi, Appl. Phys. Lett. 26 (1975) 165.

[4] J.Y. Tsao, S.T. Picraux, P.S. Peercy, M.O. Thompson, Appl. Phys. Lett. 48 (1986) 278.

[5] C.A. MacDonald, A.M. Malvezzi, F. Spaepen, J. Appl. Phys. 65 (1989) 129.

[6] H.A. Atwater, J.A. West, P.M. Smith, M.J. Aziz, J.Y. Tsao, P.S. Peercy, M.O. Thompson, Mat. Res. Soc. Symp. Proc. 157 (1990) 369.

[7] G.E. Jellison, D.H. Lowndes, Appl. Phys. Lett. 47 (1985) 718.

[8] M. Otter, Z. Phys. 161 (1961) 539. 